\title{
Oral delivery of capsaicin using MPEG-PCL nanoparticles
}

\author{
Wei PENG ${ }^{1}$, Xin-yi JIANG ${ }^{1}$, Yuan ZHU ${ }^{1}$, E Omari-Siaw ${ }^{1}$, Wen-wen DENG ${ }^{1}$, Jiang-nan $\mathrm{YU}^{1}$, Xi-ming XU ${ }^{1, *}$, Wei-ming ZHANG ${ }^{2, *}$ \\ ${ }^{1}$ Department of Pharmaceutics, School of Pharmacy, Center for Nano Drug/Gene Delivery and Tissue Engineering, Jiangsu University, \\ Zhenjiang 212013, China; ${ }^{2}$ Nanjing Institute for Comprehensive Utilization of Wild Plants, Nanjing 210042, China
}

\begin{abstract}
Aim: To prepare a biodegradable polymeric carrier for oral delivery of a water-insoluble drug capsaicin (CAP) and evaluate its quality. Methods: CAP-loaded methoxy poly (ethylene glycol)-poly( $\varepsilon$-caprolactone) nanoparticles (CAP/NPs) were prepared using a modified emulsification solvent diffusion technique. The quality of CAP/NPs were evaluated using transmission electron microscopy, powder X-ray diffraction, differential scanning calorimetry and Fourier transform infrared techniques. A dialysis method was used to analyze the in vitro release profile of CAP from the CAP/NPs. Adult male rats were orally administered CAP/NPs (35 mg/kg), and the plasma concentrations of CAP were measured with a validated HPLC method. The morphology of rat gastric mucosa was studied with HE staining.

Results: CAP/NPs had an average diameter of $82.54 \pm 0.51 \mathrm{~nm}$, high drug-loading capacity of $14.0 \% \pm 0.13 \%$ and high stability. CAP/NPs showed a biphasic release profile in vitro: the burst release was less than $25 \%$ of the loaded drug within $12 \mathrm{~h}$ followed by a more sustained release for $60 \mathrm{~h}$. The pharmacokinetics study showed that the mean maximum plasma concentration was observed $4 \mathrm{~h}$ after oral administered of CAP/NPs, and approximately $90 \mathrm{ng} / \mathrm{mL}$ of CAP was detected in serum after $36 \mathrm{~h}$. The area under the curve for the CAP/NPs group was approximately 6-fold higher than that for raw CAP suspension. Histological studies showed that CAP/NPs markedly reduced CAP-caused gastric mucosa irritation.

Conclusion: CAP/NPs significantly enhance the bioavailability of CAP and markedly reduce gastric mucosa irritation in rats.
\end{abstract}

Keywords: MPEG-PCL; nanoparticles; capsaicin; pharmacokinetics; gastric mucosa irritation

Acta Pharmacologica Sinica (2015) 36: 139-148; doi: 10.1038/aps.2014.113; published online 1 Dec 2014

\section{Introduction}

Capsaicin (CAP), an active ingredient in hot peppers of the genus Capsicum, has a variety of pharmacological activities in the cardiovascular and nervous systems ${ }^{[1,2]}$. Clinically, CAP is indicated in lumbago and sciatica and exhibits various degrees of inhibition against some food-borne pathogenic bacteria such as Listeria monocytogenes, Staphylococcus aureus, Salmonella typhimurium, and Bacillus cereus ${ }^{[1]}$. Importantly, several studies have demonstrated that CAP could effectively inhibit the growth of colorectal cancer and tumor formation through a variety of mechanisms including p53, TRPV6, and E2 $\mathrm{F}^{[3,4]}$. The clinical utilization of CAP is handicapped by rapid first-pass metabolism and a short half-life (less than $8 \mathrm{~min}$ ) after intravenous administration ${ }^{[1]}$. Furthermore, its poor aqueous solubility has led to poor oral absorption and low bioavailability

\footnotetext{
* To whom correspondence should be addressed.

E-mail xmxu@ujs.edu.cn (Xi-ming XU); botanyzh@163.com (Wei-ming ZHANG)

Received 2014-06-08 Accepted 2014-08-12
}

whether administered orally or intravenously. Currently, the usage of CAP is limited to transdermal delivery or as a skin permeation enhancer.

Poly(E-caprolactone) (PCL), approved by the United States Food and Drug Administration (FDA) as a biomedical material, has been extensively used in biodegradable pharmaceutical applications. Compared with other biodegradable polyesters, PCL exhibits lower cost, better biocompatibility and higher safety attributes. PCL can be readily loaded with a variety of hydrophobic drugs within the polymer bulk and avoids particle uptake by phagocytic cells in the liver or spleen $^{[5,6]}$. The application of PCL, however, is limited by its slow degradation rate in vivo. Grafting PCL to other watersoluble polymers has been widely reported to enhance its biodegradation rate. Methoxy poly(ethylene glycol) (MPEG)based modification has attracted increasing attention in the field of drug delivery due to its relatively low melting point that enables it to be fabricated by existing melt processing techniques and the ability to stabilize the delivery vehicle against undesirable aggregation and non-specific electrostatic 
interactions with its surroundings ${ }^{[7]}$. Furthermore, PEG segments also counteract protein absorption, which increase the circulatory half-life in the body ${ }^{[8-10]}$ due to its chain flexibility, high surface density and the absence of functional groups. Thus, in the present work, the amphiphilic matrix of methoxy poly(ethylene glycol)-poly( $\varepsilon$-caprolactone) (MPEG-PCL) containing a high percentage of PEG segments was synthesized via ring opening polymerization ${ }^{[1]}$ and used for the delivery of CAP for the first time.

Additionally, a simple, rapid, sensitive and reliable highperformance liquid chromatography (HPLC) method was established for determining pharmacokinetic parameters after oral administration in rats. Calibration curves with $R^{2}=0.994$ were constructed over a concentration range of $80-2000$ $\mathrm{ng} / \mathrm{mL}$. Therefore, in the present study, we focused on the development of CAP-loaded MPEG-PCL nanoparticles as an oral formulation aimed at improving solubility and bioavailability and reducing the side effects of CAP.

\section{Materials and methods Materials}

Methoxy poly(ethylene glycol) $(\mathrm{Mn}=2000)$ and $\varepsilon$-caprolactone and were obtained from Jenkem Technology Co, Ltd (Beijing, China) and Adamas Corporation (Shanghai Local Agent, China), respectively. CAP (99.5\% purity) was purchased from Yuanmu Biotechnology Co, Ltd (Shanghai, China). Double distilled water was produced using a Millipore water purification system (Millipore Corporation, USA) and stannous octoate $\left[\mathrm{Sn}(\mathrm{Oct})_{2}\right.$, Jenkem] was distilled prior usage. Acetonitrile and methanol used as mobile phase in HPLC were obtained from Sinopharm Chemical Reagent Co, Ltd (Shanghai, China). All other chemicals are reagent grade or higher and were used without further purification.

\section{Synthesis and characterization of MPEG-PCL block copolymers}

MPEG-PCL copolymer with a designed molecular weight of $14000 \mathrm{~g} / \mathrm{mol}$ was synthesized via ring-opening polymerization with some modification of a previously reported method ${ }^{[11]}$. Predetermined amounts of MPEG and $\varepsilon$-caprolactone were put in a dried three-necked flask containing stannous octoate $\left[\mathrm{Sn}(\mathrm{Oct})_{2}\right]$ dissolved in anhydrous toluene solution. The reaction system was refluxed under dry nitrogen atmosphere at $130^{\circ} \mathrm{C}$ for $24 \mathrm{~h}$. The crude copolymer was dissolved in dichloromethane (DCM) followed by evaporation of the toluene solution. The solution was then added to cold diethyl ether (DEE) to eliminate the unreacted MPEG. Subsequently, the resultant block copolymer was dried to a constant weight in a vacuum oven at room temperature and stored in air-tight bags pending characterization.

The formation of MPEG-PCL was determined by proton nuclear magnetic resonance ( ${ }^{1} \mathrm{H}$ NMR) and gel permeation chromatography (GPC). ${ }^{1} \mathrm{H}$ NMR spectrum was obtained using a Bruker AVANCE 600 spectrometer (Bruker, Germany) with deuterated chloroform as the solvent. In addition, the number average molecular weights $(\mathrm{Mn})$, the weight average molecular weight $(\mathrm{Mw})$ and the polydispersity index (PDI) were confirmed via a gel permeation chromatography (Waters E2695) system equipped with a refractive index detector and a Styragel HR column. HPLC-grade tetrahydrofuran (THF) was introduced as the eluent at a flow rate $1.0 \mathrm{~mL} / \mathrm{min}$. The calibration curve was generated using polystyrene standards with a molecular weight range from 900 to $30000 \mathrm{~g} / \mathrm{mol}$.

\section{Preparation of CAP-loaded MPEG-PCL nanoparticles}

CAP-loaded MPEG-PCL nanoparticles were prepared via emulsion solvent evaporation with minor modification according to the procedure reported by Zhang et al ${ }^{[12]}$. MPEG-PCL $(25 \mathrm{mg})$ and different amounts of CAP were dissolved in 1 $\mathrm{mL}$ of dichloromethane. Next, dichloromethane was carefully added to $8 \mathrm{~mL}$ of sodium cholate solution $(0.8 \%, w / v)$ and then sonicated at $600 \mathrm{~W}$ in an ice bath using a probe sonicator (XinZhi Biotechnology Co Ltd, China). The oil-in-water (o/w) emulsion formed was vigorously stirred with a magnetic stirrer (100 rounds per minute at room temperature) for $12 \mathrm{~h}$ to evaporate the solvent thoroughly. During the process of evaporation, the rationally designed copolymer self-assembled to form polymer nanoparticles with an inner hydrophobic core, which facilitated the loading of CAP in the aqueous solution. Subsequently, nanoparticles were gently washed twice with distilled water to remove free CAP on the surface followed by centrifugation (14000 rounds per minute, $4^{\circ} \mathrm{C}$ for $40 \mathrm{~min}$ ). After discarding the supernatant, $\mathrm{CAP} / \mathrm{NPs}$ were resuspended in $1 \mathrm{~mL}$ of phosphate-buffered saline (PBS; pH 7.4, $0.1 \mathrm{~mol} / \mathrm{L}$ ) and then stored at $4{ }^{\circ} \mathrm{C}$ for further use. Blank NPs were also prepared with the same method at room temperature.

\section{Drug loading and entrapment efficiency}

A Shimadzu HPLC Instrument system (Shimadzu, SCL-10A, Japan) equipped with a UV-detector (Shimadzu SPD-10A, Japan) was used for CAP quantification. An external method was used to determine the drug-loading rate of the CAP/NPs. Chromatographic analysis was performed in isocratic mode using a reversed phase C18 column $(4.6 \mathrm{~mm} \times 150 \mathrm{~mm}$, particles size $5 \mu \mathrm{m}$, Waters, USA) and the detection wavelength was $280 \mathrm{~nm}$. A methanol-water mixture $(70: 30, v / v)$ was used as mobile phase at flow rate of $1 \mathrm{~mL} / \mathrm{min}$ at $30^{\circ} \mathrm{C}$ and sample solution was injected at a volume of $20 \mu \mathrm{L}$. The HPLC was calibrated with standard solutions of $5-100 \mu \mathrm{g} / \mathrm{mL}$ of CAP dissolved in methanol (correlation coefficient of $R^{2}=0.994$ ) and the low limit of detection was $30 \mathrm{ng} / \mathrm{mL}$. The relative standard deviations (\%RSD) were all less than $5.6 \%$. The drug loading (1) and encapsulation efficiencies (2) were calculated based on the following equations:

$$
\begin{gathered}
\mathrm{DL} \%=\frac{\text { Amount of CAP in NPs }}{\text { Amount of the feeding polymer and CAP }} \times 100 \% \\
\mathrm{EE} \%=\frac{\text { Amount of CAP in NPs }}{\text { Amount of feeding CAP }} \times 100 \%
\end{gathered}
$$

\section{Particle size and surface morphology}

The average particle size and zeta potential of CAP/NPs and blank NPs were investigated by dynamic light scattering 
(DLS) with a Malvern Zetasizer (Malvern Instruments, UK) operating at a scattering angle of $90^{\circ}$ at $25^{\circ} \mathrm{C}$. In brief, each fresh sample was diluted to the appropriate concentration to ensure free diffusion and unhindered Brownian motion of the particles ${ }^{[5]}$ and was transferred into a quartz cuvette. All results were calculated from the measurement performed in triplicate and were expressed as the mean size \pm standard deviation. The morphology of prepared NPs was observed with a transmission electron microscope (TEM) (JEM-2100, JEOL, Japan). Two drops of CAP/NPs placed on a copper grid with a nitrocellulose covering were stained with phosphotungstic acid $(2 \%, w / v)$ and left to dry at room temperature. Particles were then evaluated after scanning under the microscope.

\section{FT-IR spectroscopy analysis}

The FT-IR spectra of pure drug, the physical mixture, polymers, blank NPs and drug-loaded NPs were obtained using a Nicolet AVATAR-370 FT-IR spectrophotometer (Thermo Electron Corporation, USA) at room temperature. In brief, each sample was thoroughly mixed with powdered $\mathrm{KBr}$ and compressed to a disc with pressure of 7 MPa before the FT-IR analysis. The IR spectra were obtained in the wavelength range of $4000-600 \mathrm{~cm}^{-1}$ using a resolution of $4 \mathrm{~cm}^{-1}$ and 32 scans were performed for each spectrum.

\section{X-ray diffraction}

Crystallographic assays of pure drug, the physical mixture, polymers, blank NPs and drug-loaded NPs were conducted using a powder X-ray diffractometer (XRD; D8-ADVANCE, Bruker, Germany) with a $\mathrm{Cu} K a$ radiation source at $40 \mathrm{kV}$ and $20 \mathrm{~mA}$. Before the analysis, each sample was pressed into a thin film with a Compression Molding Machine and then placed in a steel holder. The scanning range of $2 \theta$ was from $5^{\circ}$ to $60^{\circ}$ at a rate of $7^{\circ} / \mathrm{min}$.

\section{Differential scanning calorimetry}

The thermal properties of pure drug, the physical mixture, polymers, blank NPs and drug-loaded NPs were characterized by differential scanning calorimetry (DSC; PerkinElmer, USA). Each sample was placed into standard Al-crucible pans prior to accurately weighing and were then heated at rate of $10^{\circ} \mathrm{C} / \mathrm{min}$ over $30^{\circ} \mathrm{C}$ to $100^{\circ} \mathrm{C}$. Ultra-high pure nitrogen was used at a flow of $20 \mathrm{~mL} / \mathrm{min}$.

\section{Stability of nanoparticles}

Stability of nanoparticles was evaluated by measuring size variation and zeta potential measurement. Namely, the nanoparticles dissolved in PBS were stored at different temperatures and ambient humidity conditions $\left(4^{\circ} \mathrm{C} / 65 \% \mathrm{RH}\right.$, $25^{\circ} \mathrm{C} / 65 \% \mathrm{RH}$ and $40^{\circ} \mathrm{C} / 75 \% \mathrm{RH}$ ) and evaluated for particle size, zeta potential and drug content. The samples were withdrawn at specified time intervals of $0,1,2$, and 3 months ${ }^{[13-15]}$. The changes in mean size and zeta potential were monitored by the Malvern Zetasizer operating at a scattering angle of $90^{\circ}$ at $25^{\circ} \mathrm{C}$. Drug content analysis was conducted using the validated HPLC method previously mentioned.

\section{In vitro release studies}

The in vitro release behavior of CAP/NPs into two release media was investigated by a dialysis method over $72 \mathrm{~h}^{[16]}$. In brief, CAP/NPs solution placed and sealed in dialysis bags (MWCO $=3500 \mathrm{Da}$, Greenbird Inc, Shanghai, China) was incubated in $100 \mathrm{~mL}$ of the release media at $37^{\circ} \mathrm{C}$ under vigorous shaking (100 rounds per minute) for $72 \mathrm{~h}$. The release media, simulated gastric fluid (SGF; $\mathrm{pH} 1.2 ; 0.1 \mathrm{~mol} / \mathrm{L})$ or PBS (pH 7.4; $0.1 \mathrm{~mol} / \mathrm{L})$, contained Tween- $80(0.3 \%, w / v)$ to assure sink conditions. At discrete time intervals $(0.25,0.5,1,2,4,6,8,12$, $24,36,48,60$, and $72 \mathrm{~h}$ ), $0.5 \mathrm{~mL}$ of release media was drawn and replenished with an equal volume of fresh media. Then, samples were centrifuged (10000 rounds per minute for 10 $\mathrm{min}$ ) to separate the released drug from the nanoparticulate $\mathrm{CAP}^{[17,18]}$. The concentration of CAP in the removed supernatant was determined using the HPLC system. CAP released from the stock solution was set as control. The results were the mean of three test runs and all values were expressed as the mean \pm standard deviation. To evaluate the possible release mechanism, zero-order, first-order, Higuchi, and Weibull models were used to fit the in vitro drug release pattern. The fitting to different release models was conducted using Microsoft office Excel $^{\oplus}$ and the final curves were accomplished using Originlab $^{\circledast}$ 8.0. The Weibull model is represented by the following equation (3):

$$
\operatorname{Ln} \ln \left(\frac{1}{1-M}\right)=a \ln t+b
$$

where $M$ is the accumulated release rate and $t$ is time; a and $\mathrm{b}$ are constant. The goodness of fitting for the release kinetic models was evaluated with correlation coefficient values $\left(R^{2}\right)$.

\section{Preparation of test animals}

The study protocol was approved by the Ethics Committee of Jiangsu University. Ten male Sprague-Dawley (SD) rats weighing 250-300 g were purchased from the Animal Centre of Jiangsu University and randomly divided into two groups $(n=5)$ for pharmacokinetic investigation. Animals were starved overnight $(12 \mathrm{~h})$ with free access to water before dosing to avoid drug-food interactions. CAP/NPs were orally administered to one group and the other group received crude drug dispersed in $0.5 \%$ CMC-Na solution at the CAP dose of $35 \mathrm{mg} / \mathrm{kg}$ body weight. Blood samples of approximately 500 $\mu \mathrm{L}$ were collected via the orbital veins into heparinized polyethylene tubes at scheduled time points $(0.25,0.5,1,2,4,6,8$, 12,24 , and $36 \mathrm{~h}$ ) and centrifuged at 3000 rounds per minute for $10 \mathrm{~min}$ to separate plasma. Then, the supernatant plasma fraction was transferred into a clear glass tube. Harvested plasma samples were stored at $-70^{\circ} \mathrm{C}$ in an ultra-low temperature freezer until further analysis.

\section{Plasma sample preparation}

The CAP in serum was extracted by the Liquid-Liquid extraction method (LLE). After $200 \mu \mathrm{L}$ of plasma was mixed with $400 \mu \mathrm{L}$ doubled distilled water in a 10-mL glass tube, an aliquot of $50 \mu \mathrm{L}$ internal standard (IS; 1-Naphthol) working solu- 
tion and $500 \mu \mathrm{L}$ acetonitrile were successively added to the mixture. The resulting sample was vortex-mixed vigorously for $1 \mathrm{~min}$ to precipitate plasma proteins followed by adding 3 $\mathrm{mL}$ of a cyclohexane-ethyl acetate mixture $(1: 1, v / v)$. The resultant mixture was subjected to centrifugation at 3000 rounds per minute for $10 \mathrm{~min}$. Then, the upper layer was transferred into a tube and evaporated to dryness under a gentle stream of nitrogen gas at $40^{\circ} \mathrm{C}$. The residue was reconstituted with $100 \mu \mathrm{L}$ of methanol and centrifuged at 3000 rounds per minute for $5 \mathrm{~min}$. A $20 \mu \mathrm{L}$ aliquot of the supernatant was injected into the HPLC system for quantitative analysis.

\section{Validation of HPLC Method}

An internal method was applied to determine the amount of CAP in plasma. Therefore, two different HPLC methods were employed for the drug loading and in vivo experiments. The HPLC separation was performed on an Inertsil ODS-SP analytical column $(4.6 \mathrm{~mm} \times 150 \mathrm{~mm}, 5 \mu \mathrm{m}$, Shimadzu, Japan). The system operated under an isocratic flow rate of $1 \mathrm{~mL} / \mathrm{min}$ using a mobile phase of acetonitrile-water $(43: 57, v / v)$, which was filtered through a membrane $(0.22 \mu \mathrm{m})$ and degassed in a sonicator bath for $30 \mathrm{~min}$ prior to usage. The injection volume for HPLC analysis was set at $20 \mu \mathrm{L}$ and the temperature of column was maintained at $50^{\circ} \mathrm{C}$ during analysis. A calibration curve $(y=0.002 x-0.0154)$ was constructed using six calibration standards that showed excellent linearity over the concentration range of $80-2000 \mathrm{ng} / \mathrm{mL}$ (correlation coefficient, $\left.R^{2}=0.994\right)$. The accuracy and precision of the assay were determined by three replicates of spiked plasma samples at low, medium and high concentrations $(125,1000$, and $2000 \mathrm{ng} / \mathrm{mL})$. The extraction recoveries were in the range of $80.5 \%-86.4 \%$. The limit of detection (LOD) ${ }^{[19]}$ of this assay was $30 \mathrm{ng} / \mathrm{mL}$ at a signal-to-noise ratio $(\mathrm{S} / \mathrm{N})$ of $>3$ and the lowest limit of quantification (LOQ) determined was $75 \mathrm{ng} / \mathrm{mL}$ (S/N ratio $>10$ ) with a $\%$ RSD of $<15 \%$. Accuracy was calculated using equation (4):

$$
\text { Accuracy }=\frac{\text { Mean measured concentration }}{\text { Nominal concentration }} \times 100 \%
$$

The intra- and inter-day precision was expressed as the relative standard deviation and the results were obtained via analysis of samples on three separate days.

\section{Analysis of Pharmacokinetic parameters}

Pharmacokinetic parameters of the drug, namely, peak plasma concentration $\left(C_{\max }\right)$, time to $C_{\max }\left(T_{\max }\right)$, mean residence time (MRT) and area under the concentration-time curve $\left(\mathrm{AUC}_{\mathrm{t}}\right)$ were calculated using the BAPP 2.3 pharmacokinetic software (purchased from the Center of Drug Metabolism of China Pharmaceutical University, China ${ }^{[20]}$. All parameter values are reported as the mean value \pm standard deviation unless otherwise noted. The relative bioavailability (F) after administration was determined by calculating the ratio of AUC for the test formulation $\left(\mathrm{AUC}_{\mathrm{T}}\right)$ and $\mathrm{AUC}$ for the reference formulation $\left(\mathrm{AUC}_{\mathrm{R}}\right)$ according to the following equation (5):

$$
\mathrm{F} \%=\frac{\mathrm{AUC}_{\mathrm{T}}}{\mathrm{AUC}_{\mathrm{R}}} \times 100 \%
$$

\section{In vitro-in vivo correlation}

In vitro-in vivo correlation (IVIVC) refers to the predictive mathematical model describing the relationship between in vitro dissolution and in vivo input rate ${ }^{[21]}$. Although four categories of IVIVCs have been described in the FDA guidance, a level A correlation model is used to describe the relationship because that is considered most informative and very useful from a regulatory viewpoint. In addition, CAP, a poorly water-soluble drug, exhibits low solubility and low permeabil${ }^{i t y}{ }^{[22]}$. The results of the fraction absorbed (FRA) and fraction of drug dissolved (FRD) were obtained using the method of Wagner-Nelson using the BAPP software. The relationship between FRA and FRD was fitted using the nonlinear regression model as the following equation (6):

$$
Y=a+b X+c X^{2}
$$

where $a, b, c$ represent regression parameters of the equation, $Y$ is the FRA in vivo and $X$ is the FRD in vitro. The IVIVC was evaluated by the correlation coefficient $\left(R^{2}\right)$.

\section{Gastric mucosa irritation test}

Nine male SD rats weighting $300 \pm 20 \mathrm{~g}$ were randomly divided into three groups. After $12 \mathrm{~h}$ of fasting, one group was treated with physiological saline as control and the other two groups were orally gavaged with free CAP (suspended in 0.5\% CMCNa solution) or NPs at the CAP dose of $35 \mathrm{mg} / \mathrm{kg}$. Two hours later, stomachs were removed, cut open along the greater curvature and rinsed thoroughly with physiological saline. Subsequently, the samples were persevered in 10\% formalin. Sections $(3-4 \mu \mathrm{m})$ of tissue were cut using a microtome and stained with hematoxylin and eosin (H\&E) before microscopic examination.

\section{Results}

\section{Characterization of the amphiphilic copolymer}

Figure 1 shows the ${ }^{1} \mathrm{H}$ NMR spectrum of the MPEG-PCL block copolymer. The signal at $\delta 3.649 \mathrm{ppm}$ was assigned to the $-\mathrm{CH}_{2}$ protons of the PEG chain. In addition, the peaks at $\delta 2.294-2.319,1.619-1.678,1.384-1.410$, and $4.050-4.072$ ppm were attributed to the various methylene protons of the PCL units corresponding to the results reported in the literature ${ }^{[23,24]}$. The Mn obtained from ${ }^{1} \mathrm{H}$ NMR was calculated to be approximately $16000 \mathrm{~g} / \mathrm{mol}$ by comparing the peak intensity at approximately 2.31 with $3.65 \mathrm{ppm}$. The $\mathrm{Mn}$ and $\mathrm{Mw}$ detected from the GPC were summarized in Table 1. Notably, GPC chromatographs from the block copolymer showed a unimodal shape and narrow molecular weight distributions.

\section{Characterization of nanoparticles}

A drug loading content of $14.0 \% \pm 0.13 \%$ and encapsulation efficiency of $81.5 \% \pm 0.9 \%$ were obtained using a CAP feeding ratio of $20 \%$. As observed in Figure 2A, the prepared CAP/NPs exhibited a narrow size distribution with an average diameter 


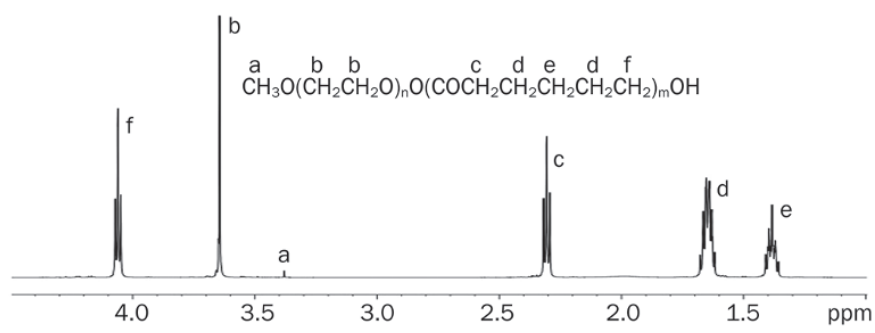

Figure 1. ${ }^{1} \mathrm{H}$ NMR spectrum of MPEG-PCL dissolved in $\mathrm{CDCl}_{3}$.

Table 1. Characterization of MPEG-PCL copolymer.

\begin{tabular}{lccccc}
\hline $\begin{array}{c}\text { MPEG/PCL } \\
\text { (theoretical) }\end{array}$ & $\begin{array}{c}\text { Total Mn } \\
\text { (theoretical) }\end{array}$ & $\begin{array}{c}\text { Total Mn } \\
(\text { GPC })\end{array}$ & $\begin{array}{c}\text { Total Mw } \\
(\text { GPC })\end{array}$ & $\begin{array}{c}\text { PDI } \\
(\text { GPC })\end{array}$ & $\begin{array}{r}\text { Total Mn } \\
\left({ }^{1} \mathrm{H} \mathrm{NMR}\right)\end{array}$ \\
\hline $2000 / 12000$ & 14000 & 10595 & 14283 & 1.35 & 16000 \\
\hline
\end{tabular}

of $82.54 \pm 0.51 \mathrm{~nm}$ and a low polydispersity index (PDI) in the range of $0.09 \pm 0.02$. In addition, the zeta potential of CAP/NPs was found to be $-24.67 \pm 4.3 \mathrm{mV}$, indicating the high stability of NPs.

The morphology of NPs prepared using the modified emulsification solvent diffusion method is shown in Figure 2B. According to the TEM images, the prepared CAP/NPs had a spherical appearance with an approximate average diameter of $80 \mathrm{~nm}$, which was consistent with the results determined by dynamic light scattering technique.

As shown in Figure 3A, typical absorption peaks of the car-
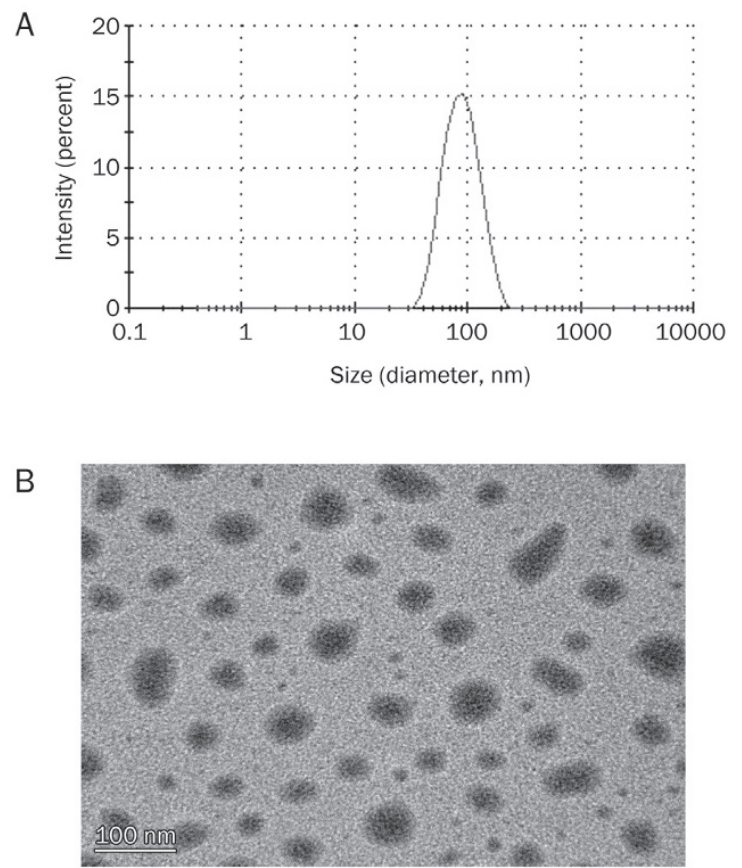

Figure 2. Particle size and size distribution of CAP/NPs by DLS (A); TEM image of prepared CAP/NPs (B), the bar is $100 \mathrm{~nm}$. bonyl groups $(\mathrm{C}=\mathrm{O})$ of PCL appeared at approximately 1725 $\mathrm{cm}^{-1}$ and the peaks at $2875 \mathrm{~cm}^{-1}$ and $2937 \mathrm{~cm}^{-1}$ were assigned to the stretching vibration of $\mathrm{C}-\mathrm{H}$ bond of MPEG and PCL. The spectrum of the blank NPs was similar to that of the copolymer because they are characterized by the bands described above. Three characteristic absorbance bands of raw CAP were clearly observed at approximately 1600, 2922, and 3278 $\mathrm{cm}^{-1}$. These bands were also found in the physical mixture. The remaining peaks in the mixture were attributed to the induction of MPEG-PCL, which was confirmed by comparing of the spectra of the mixture with that of the copolymer. Moreover, in the case of fingerprint regions, there were obvious differences between the mixture and NPs. The fingerprint regions of the NPs were similar to the copolymer, whereas the mixture exhibited a CAP-like fingerprint.

To elucidate potential changes of drug crystals in the nanoparticles formulation, X-ray powder diffraction (XRD) was performed and the spectra of CAP, CAP/NPs, MPEGPCL, blank NPs and the physical mixture were shown in Figure 3B. Raw CAP material is crystalline in nature, as demonstrated by crystallographic analysis, with characteristic peaks at approximately $5.6^{\circ}, 11.5^{\circ}, 21.5^{\circ}$, and $24.6^{\circ}$. The MPEG-
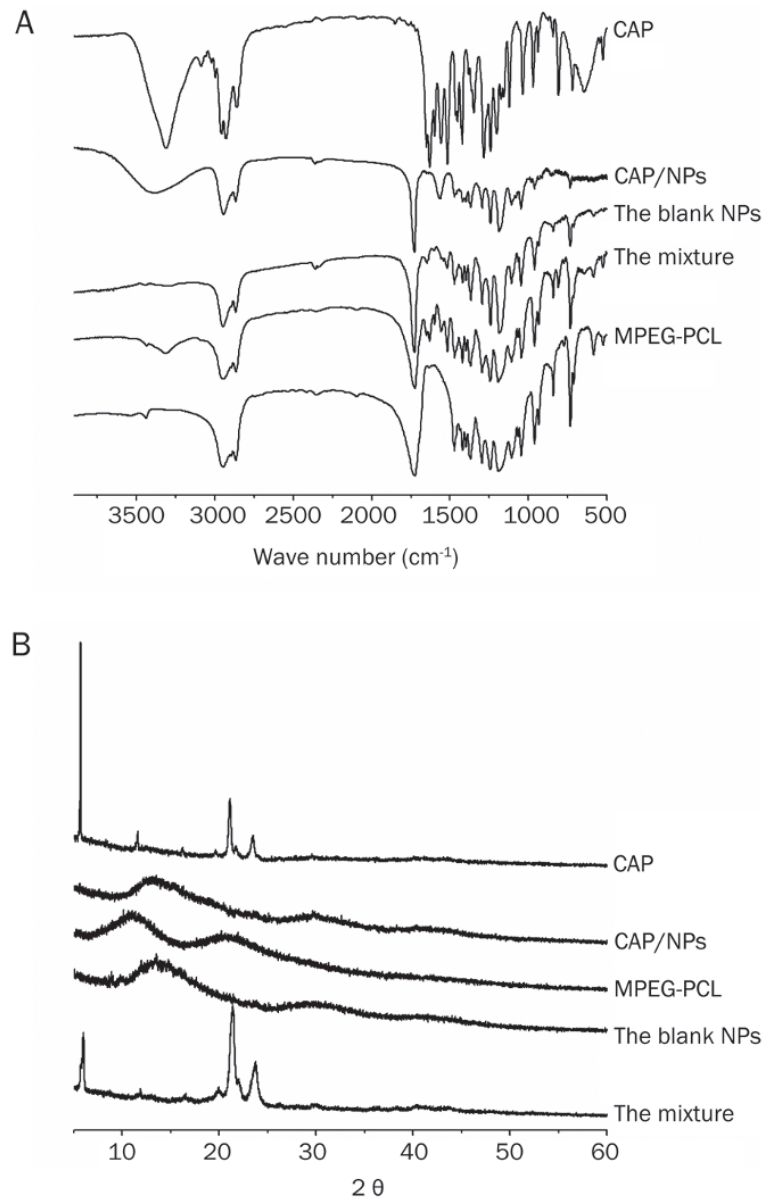

Figure 3. (A) FT-IR spectra of blank NPs powder, MPEG-PCL copolymer, lyophilized loaded MPEG-PCL, CAP and the physical mixture of free CAP and MPEG-PCL; (B) respective $\mathrm{X}$-ray diffraction spectra. 
PCL was amorphous, which was confirmed by the absence of diffraction peaks. In addition, most crystalline peaks of CAP were apparent in the spectrum of the mixtu re indicating that the physical state of drug in the mixture was not changed.

Thermal behaviors of CAP, MPEG-PCL, NPs, blank NPs and the physical mixture of CAP and the synthesized copolymer were analyzed by DSC and the results of thermal analysis are shown in Figure 4. CAP exhibits a melting peak between $63^{\circ} \mathrm{C}$ and $70^{\circ} \mathrm{C}$, centered at approximately $67^{\circ} \mathrm{C}$ as observed in the figure, which was consistent with a previous report ${ }^{[25]}$. MPEGPCL showed a melting point at approximately $61^{\circ} \mathrm{C}$ as well. It is important to mention that a marked shift in peak position was observed for the physical mixture, which is indicative of a crystalline state for CAP in the mixture. The behavior can be attributed to the interactions between $\mathrm{CAP}$ and the polymer in the solid state. For lyophilized CAP/NPs, no inflexion point at $67^{\circ} \mathrm{C}$ was measured.

\section{Stability study}

As shown in Table 2, no significant change in the mean diameter of NPs was observed after 3 months of storage at $4{ }^{\circ} \mathrm{C} / 65 \%$ $\mathrm{RH}, 25^{\circ} \mathrm{C} / 65 \% \mathrm{RH}$ and $40^{\circ} \mathrm{C} / 75 \% \mathrm{RH}$ and the particle size did not exceed $90 \mathrm{~nm}$. Additionally, the drug content of CAP/ NPs showed only minor changes at the various temperatures during 3 months. In addition, the surface charge of NPs was measured to be approximately $-24 \mathrm{mV}$. These results further confirmed that the NPs were highly stable in PBS.

\section{In vitro release of CAP/NPs}

The in vitro release behaviors of CAP/NPs in two release media are shown in Figure 5. It was clearly observed that the release profiles of CAP/NPs in SGF and PBS were statistically similar because there was no significant difference $(P>0.05)$ between the release profiles of CAP from either media. This phenomenon demonstrates that the release media, SGF or PBS, has little influence on the in vitro release behavior of the NPs. In addition, the CAP-loaded MPEG-PCL nanoparticles showed a biphasic release profile. The burst of CAP from the formulation within $12 \mathrm{~h}$ was less than $25 \%$ in either media fol-
Table 2. Percent drug remaining, diameter and zeta potential in CAP/ NPs stored at various temperatures $\left(4^{\circ} \mathrm{C} / 65 \% \mathrm{RH}, 25^{\circ} \mathrm{C} / 65 \% \mathrm{RH}\right.$, and $40^{\circ} \mathrm{C} / 75 \% \mathrm{RH}$ ) for 3 months.

\begin{tabular}{ccccc}
\hline $\begin{array}{c}\text { Time } \\
(\text { month })\end{array}$ & $\begin{array}{c}\text { Temperature } \\
(\mathrm{RH})\end{array}$ & $\begin{array}{c}\text { \%Drug } \\
\text { remained }\end{array}$ & $\begin{array}{c}\text { Diameter } \\
(\mathrm{nm})\end{array}$ & $\begin{array}{c}\text { Zeta potential } \\
(\mathrm{mV})\end{array}$ \\
\hline 0 & $4^{\circ} \mathrm{C} / 65 \%$ & 100 & $82.52 \pm 0.51$ & $-24.67 \pm 4.3$ \\
1 & $4^{\circ} \mathrm{C} / 65 \%$ & 99.67 & $84.17 \pm 0.91$ & $-23.63 \pm 5.4$ \\
2 & $4^{\circ} \mathrm{C} / 65 \%$ & 99.48 & $86.79 \pm 1.06$ & $-24.01 \pm 3.6$ \\
3 & $4^{\circ} \mathrm{C} / 65 \%$ & 99.15 & $86.46 \pm 0.72$ & $-23.45 \pm 4.6$ \\
& & & & \\
0 & $25^{\circ} \mathrm{C} / 65 \%$ & 100 & $82.52 \pm 0.51$ & $-24.67 \pm 4.3$ \\
1 & $25^{\circ} \mathrm{C} / 65 \%$ & 99.59 & $83.52 \pm 0.74$ & $-23.21 \pm 5.6$ \\
2 & $25^{\circ} \mathrm{C} / 65 \%$ & 99.32 & $86.42 \pm 0.83$ & $-22.81 \pm 5.2$ \\
3 & $25^{\circ} \mathrm{C} / 65 \%$ & 99.10 & $87.05 \pm 0.94$ & $-23.79 \pm 3.7$ \\
& & & & \\
0 & $40^{\circ} \mathrm{C} / 75 \%$ & 100 & $82.52 \pm 0.51$ & $-24.67 \pm 4.3$ \\
1 & $40^{\circ} \mathrm{C} / 75 \%$ & 99.43 & $85.36 \pm 1.13$ & $-23.15 \pm 3.5$ \\
2 & $40^{\circ} \mathrm{C} / 75 \%$ & 98.42 & $87.83 \pm 0.97$ & $-23.79 \pm 4.8$ \\
3 & $40^{\circ} \mathrm{C} / 75 \%$ & 97.76 & $87.50 \pm 0.75$ & $-24.46 \pm 5.3$ \\
\hline
\end{tabular}

lowed by a more sustained release rate for $60 \mathrm{~h}$. Compared to the nanoparticle formulation, the release profiles of the CAP stock solution had marked differences, showing its release behavior was dependent on $\mathrm{pH}$. The CAP stock solution release rate was faster in SGF than PBS. Approximately $68 \%$ of CAP was released from the stock solution in PBS within $4 \mathrm{~h}$ compared with nearly $100 \%$ in SGF. Furthermore, based on the correlation coefficient criterion, the release profiles of the NPs in SGF and PBS were best fitted with the Weibull mathematical model $\left(R^{2}=0.972\right.$ and 0.969 , respectively).

\section{In vivo pharmacokinetics}

The data from the validation of the assay method in rat plasma are presented in Table 3. The precision of intra- and inter-day were $1.16 \%-4.47 \%$ and $1.08 \%-7.91 \%$, respectively, and all values of accuracy were found to be satisfactory and in line with international criteria ${ }^{[26]}$. This suggested that the

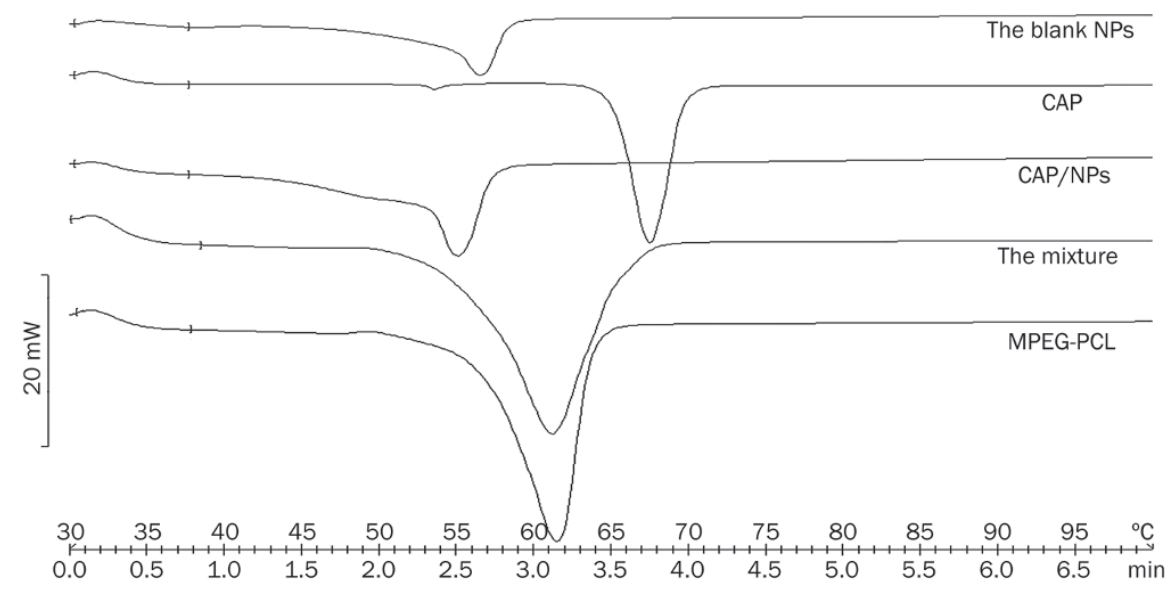

Figure 4. DSC curves of blank NPs powder, lyophilized CAP/NPs, the physical mixture of free CAP and MPEG-PCL, MPEG-PCL copolymer and pure CAP. 
A

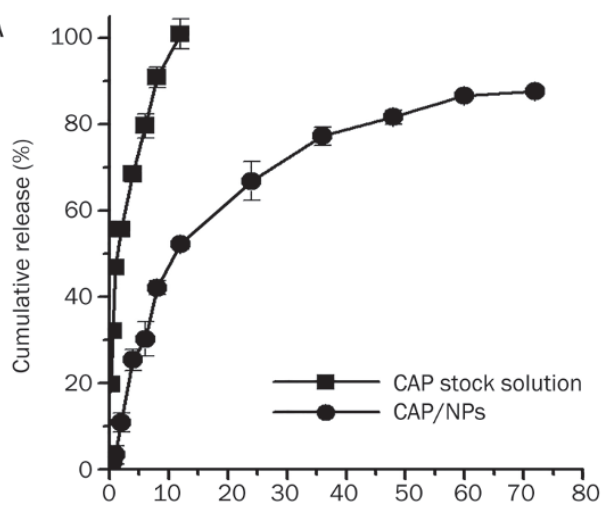

B

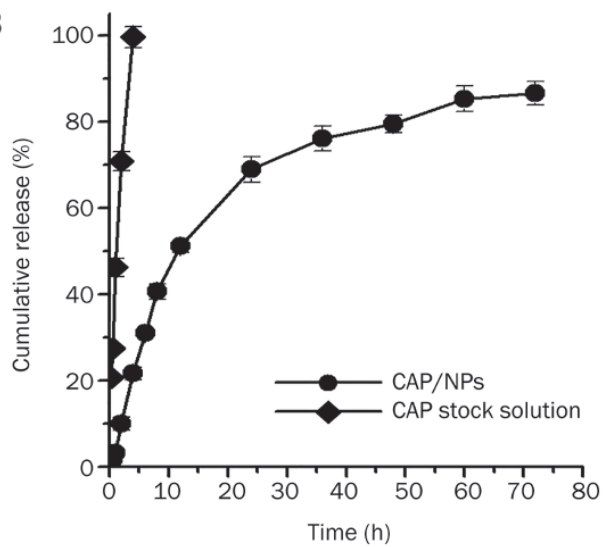

Figure 5. Drug release profiles of free CAP and CAP/NPs in PBS (pH 7.4) (A) and SGF ( $\mathrm{pH}$ 1.2) (B). Data are expressed as the mean \pm SD $(n=3)$.

method is suitable for quantitative analysis of CAP in plasma samples. Figure 6 shows the mean CAP plasma concentration versus time profiles after oral administration at the $35 \mathrm{mg} / \mathrm{kg}$ CAP dose in two formulations (raw CAP suspension and CAP loaded in MPEG-PCL nanoparticles). The relevant pharmacokinetic parameters have also been summarized in Table 4 . With regard to the single compartmental analysis, the NPs exhibited pharmacokinetic parameters that were significantly

Table 3. Intra- and inter- assay relative accuracy and precision for CAP in rat plasma $(n=6)$.

\begin{tabular}{llll}
\hline $\begin{array}{l}\text { Theoretical } \\
\text { concentration } \\
(\mathrm{ng} / \mathrm{mL})\end{array}$ & $\begin{array}{l}\text { Concentration found } \\
(\text { mean } \pm \mathrm{SD}, \mathrm{ng} / \mathrm{mL})\end{array}$ & RSD (\%) & Accuracy (\%)
\end{tabular}

\begin{tabular}{lrlr}
\hline Intra-day run & & & \\
125 & $136 \pm 6.08$ & 4.47 & 108.80 \\
1000 & $1000.33 \pm 11.59$ & 1.16 & 100.03 \\
2000 & $2032.33 \pm 59.53$ & 2.93 & 101.62 \\
& & & \\
Inter-day run & & & \\
125 & $132 \pm 10.44$ & 7.91 & 105.61 \\
1000 & $994.67 \pm 10.69$ & 1.08 & 99.47 \\
2000 & $1990 \pm 29.82$ & 1.50 & 99.50 \\
\hline
\end{tabular}

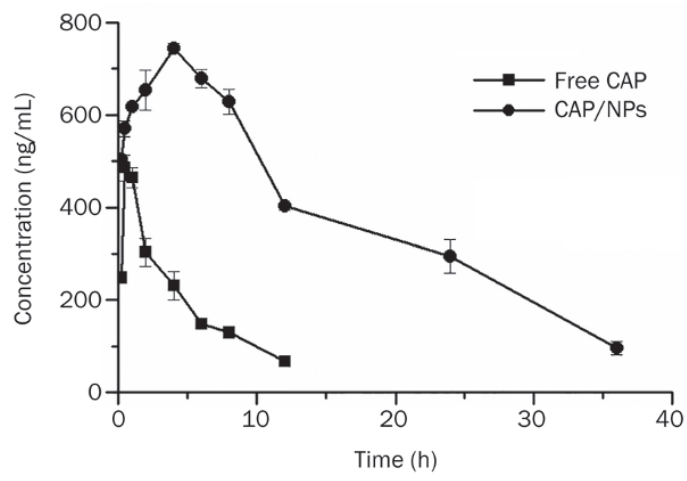

Figure 6. Pharmacokinetic profile of free CAP and CAP/NPs. Data are expressed as the mean $\pm \operatorname{SD}(n=5)$.

altered compared to those of the free CAP. After oral administration of the free CAP suspension, a maximum plasma concentration $\left(C_{\max }\right)$ of $491.94 \pm 17.89 \mathrm{ng} / \mathrm{mL}$ was reached in $0.5 \mathrm{~h}$, indicating that the drug was absorbed rapidly. Subsequently, plasma concentrations decreased sharply resulting in a high $K_{\mathrm{e}}(0.187 \pm 0.006)$ and short half-life $(3.70 \pm 0.11 \mathrm{~h})$. These results suggest that the drug was rapidly distributed and metabolized leaving a minimum drug concentration after $24 \mathrm{~h}$. By contrast, $\mathrm{CAP} / \mathrm{NPs}$ showed a markedly delayed blood clearance. Its pharmacokinetic parameters suggested that the NPs could extend the half-life of CAP from $3.70 \pm 0.11 \mathrm{~h}$ to $13.88 \pm 13.73 \mathrm{~h}$ $(P<0.01)$ and delay the MRT from $5.91 \pm 0.38 \mathrm{~h}$ to $18.02 \pm 19.91 \mathrm{~h}$ $(P<0.01)$. It is also worth noting that the $C_{\max }$ of the NPs $(743.44 \pm 10.67 \mathrm{ng} / \mathrm{mL})$ was reached within $4 \mathrm{~h}$, demonstrating that there are different absorption rates for free CAP and CAP/NPs. Clearly, the nanoparticles are effective at increasing drug absorption because the delayed $T_{\max }(4 \mathrm{~h})$ demonstrates sustained release of CAP. In addition, the $\mathrm{AUC}_{0-\mathrm{t}}$ for the formulation was approximately 6-fold higher than that of free CAP $(P<0.01)$. The relative bioavailability of CAP/NPs showed approximately 6-fold increase compared to free drug.

\section{In vitro-in vivo correlation}

Exploring the correlation between in vitro permeation and in vivo absorption is an important part in the dosage form development process because it can shorten the drug development period and economize resources ${ }^{[27]}$. The point-to-point

Table 4. Pharmacokinetic parameters of free CAP suspension and CAPloaded MPEG-PCL nanoparticles in rats after oral administration $(n=5)$.

\begin{tabular}{llcc}
\hline & & \multicolumn{2}{c}{ Formulations } \\
Parameters & Units & $\begin{array}{c}\text { Free CAP } \\
\text { suspension }\end{array}$ & $\begin{array}{c}\text { CAP MPEG-PCL } \\
\text { nanoparticles }\end{array}$ \\
\hline AUC $_{0-\mathrm{t}}$ & $\mathrm{h} \cdot \mathrm{ng} / \mathrm{mL}$ & $2324.30 \pm 113.25$ & $13848.65 \pm 186.20$ \\
$C_{\max }$ & $\mathrm{ng} / \mathrm{mL}$ & $491.94 \pm 7.89$ & $743.44 \pm 10.67$ \\
$T_{\max }$ & $\mathrm{h}$ & 0.5 & 4 \\
$t_{1 / 2}$ & $\mathrm{~h}$ & $3.70 \pm 0.11$ & $13.88 \pm 13.73$ \\
MRT & $\mathrm{h}$ & $5.91 \pm 0.38$ & $18.02 \pm 19.91$ \\
\hline
\end{tabular}


relationship between in vitro and in vivo release profiles was a level A correlation as defined by the US Food and Drug Administration $^{[28]}$. Level A correlation between in vitro dissolutions (in SGF and PBS) and in vivo absorption was constructed. Good point-to-point relationship of in vitro permeation and in vivo absorption were observed for the NPs with a correlation coefficient of 0.998 and 0.996 in SGF and PBS, respectively. It is important to note that reliable IVIVCs allow prediction of the in vivo bioavailability or bioequivalence of CAP based on the in vitro drug dissolution.

\section{Gastric mucosa irritation test}

In this investigation, it was anticipated that the CAP-loaded copolymer nanoparticles could reduce the irritation effect on the gastrointestinal tract compared with that caused by CAP administered as an aqueous suspension. As shown in Figure 7C, marked mucosal bleeding and inflammatory cell infiltrations were observed in rat stomachs after administration of pure CAP suspension. However, few or no lesions were observed in the mucosal surface of the rats that received the NPs formulation (Figure 7B).

\section{Discussion}

PCL is one of the most promising polymers for controlled and site-specific drug delivery because of its biocompatibility and good biodegradable nature ${ }^{[29]}$. It can also readily blend efficiently with many other polymers, especially grafted MPEG block to satisfy the required biophysical properties for most formulations. The amphiphilic copolymer of MPEG-PCL containing a high percentage of PEG segments was synthesized by ring opening polymerization with $\mathrm{Sn}(\mathrm{Oct})_{2}$ as the catalyst. In this study, molecular weight of the copolymer was controlled by adjusting the molar ratio of $\varepsilon-C L$ and MPEG. In addition, the critical micelle concentration of the copolymer decreased with increasing PCL length which was consistent with published literature ${ }^{[23]}$.

CAP has been extensively investigated in pharmacy and neuroscience ${ }^{[30]}$ due to its varied pharmacological activities. However, owing to its high hydrophobic structure, CAP exhibits very poor aqueous solubility, which results in formulation difficulties. Several reports have been published on the delivery of CAP using liposomes ${ }^{[31]}$ and microemulsions ${ }^{[1]}$.
These nanosized delivery systems have already proven their effectiveness in solubilizing CAP. However, there are a few drawbacks of these systems, such as high quantities of surfactants and co-surfactants, low drug loading and being unstable systems. Thus, in our study, synthesized MPEGPCL was used as the carrier of CAP for its ability to overcome the deficiencies mentioned above. We found that the solubility of CAP in water was notably improved by the MPEGPCL vehicle compared to the unformulated drug. This can be attributed to the hydrophobic core formed by the amphiphilic copolymer in the aqueous medium. The cargo space in turn loads CAP, thus allowing it to exist in the molecular or amorphous form.

Although several methods are used to prepare MPEG-PCL nanoparticles, the emulsion solvent evaporation method was adopted in this study because it is simple, reproducible, economic and widely employed ${ }^{[32]}$. EE\% and DE\% are important indexes for a drug delivery system. This is true for expensive drugs, especially those used in the treatment of cancer ${ }^{[33]}$. We observed that increased drug-to-polymer ratios resulted in marginal elevation of drug loading but a gradual decline in $\mathrm{EE} \%$. However, no obvious difference was found in particle size and PDI, which was consistent with a previous report ${ }^{[34]}$. In our work, the mean droplet size of the prepared nanoparticles was approximately $80 \mathrm{~nm}$ with a narrow distribution. Many related studies have shown that the particle size and distribution (PDI) of the NPs are very important factors for systemic drug uptake. Smaller particle sizes $(<200 \mathrm{~nm})$ and narrow distribution range decrease the rate of mononuclear phagocyte system uptake and tend to accumulate in tumor sites ${ }^{[35,36]}$. As such, the relatively small particle size and narrow PDI of CAP/NPs could allow for the accumulation of more CAP at the site of action thereby eliciting a sustained effect. According to TEM images, the prepared CAP/NPs had a spherical appearance with an approximate average diameter of $80 \mathrm{~nm}$, which was consistent with the result obtained by DLS. In addition, it was clearly observed that no drug crystals surrounded the NPs, suggesting that the preparation process was free from drug crystallization. The MPEG-PCL spectrum was also in line with previous reports ${ }^{[7,24]}$, further implying that MPEG-PCL was successfully synthesized. There was little or no interaction between the drug and carrier demon-
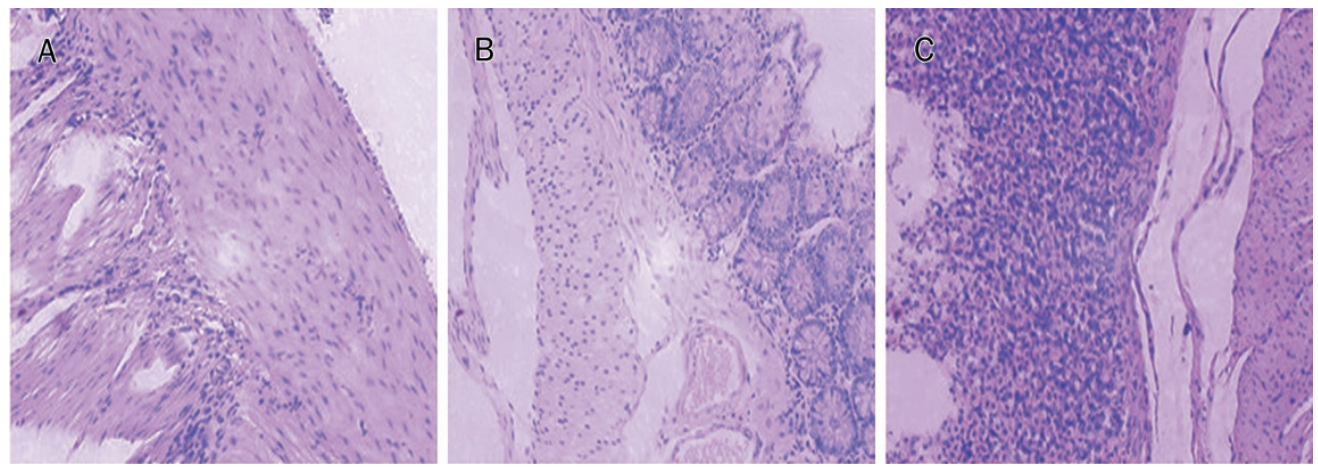

Figure 7. Histological examination for gastric mucosa of rats after administration of normal saline (A), CAP/NPs (B), and free CAP (C). 
strated by the lack of a new absorption peak from the FT-IR spectra. Additionally, due the absence of crystalline peaks in the pattern of $\mathrm{CAP} / \mathrm{NPs}$, CAP can be said to be molecularly dispersed in the copolymer. These results were further supported by data obtained from DSC measurements.

Formulation stability plays important roles in drug development procedures, long-term storage and transportation. The stability of CAP/NPs was studied by storing NPs in PBS at different temperature for three months. There was no significant change in the mean diameter or the surface charge of NPs over the period or the drug content. This fact may be attributed to the PEG chains on the surface of the nanoparticles, which prevent the aggregation of CAP/NPs. Compared to the CAP stock solution, the in vitro release of CAP/NPs in both media showed a relatively slower release rate, possibly due to the effect of both drug diffusion and polymer swelling.

Following oral administration, the bioavailability of CAPloaded MPEG-PCL was significantly improved compared to CAP in aqueous suspension. $C_{\max }$ and AUC are important features of the blood level profile. AUC depicts the extent of drug absorption. We observed that both AUC and $C_{\max }$ of $\mathrm{CAP} / \mathrm{NPs}$ were significantly greater than the control group. These results could be due to the behavior of the copolymer nanoparticles, which reduce drug uptake by plasma proteins and the mononuclear phagocyte system while protecting CAP from degradation. CAP has been used for the treatment of chronic musculoskeletal or neuropathic pain for many years ${ }^{[37]}$. Its range of clinical usage is often limited due to the incidence of gastrointestinal irritation and hemorrhage. CAP/NPs, in this study, visibly reduced the irritation effect on the gastrointestinal tract compared with the aqueous suspension. The reduced direct contact of CAP with the surface of gastrointestinal tract mucosa offered by the protective effect of the carrier could account for this phenomenon. These results, therefore, establish that the amphiphilic copolymer can significantly decrease the gastric mucosa irritation side-effect of CAP.

In the present study, the CAP nanoparticles based on the MPEG-PCL copolymer were fabricated by the solvent emulsification/evaporation technique where particles with small size, uniform distribution and higher encapsulation efficiency were successfully produced. The nanoparticle formulation was characterized by TEM, XRD, DSC, and FT-IR techniques. The in vitro release behavior of the NPs exhibited a sustained release of $\mathrm{CAP}$, suggesting that the release behavior of the NPs was governed by MPEG-PCL. The results of histological studies showed that CAP entrapped by the copolymer can markedly reduce irritation in the gastric mucosa in rats. Furthermore, after oral administration of $35 \mathrm{mg} / \mathrm{kg}$ CAP as a NPs formulation and free CAP, plasma concentrations of CAP were detected by a validated HPLC method. The NPs have the potential for significantly lowering the clearance of CAP and increasing its bioavailability compared with the control formulation. Based on these results, it can be concluded that the MPEG-PCL nanoparticles investigated in this study may be a good drug delivery vehicle for CAP.

\section{Acknowledgements}

This work was supported by the National Natural Science Foundation of China (30973677), National "Twelfth Five-Year" Plan for Science \& Technology Support (2012BAD36B01), the Doctoral Fund of Ministry of Education of China (20113227110012), Special Funds for 333 (BRA2013198) and 331 Projects and Industry-University-Research Institution Cooperation (JHB2012-37, GY2012049, and GY2011028) in Jiangsu Province and Zhenjiang City. The authors also thank the Jiangsu University Ethics Committee for their guidance in the animal experiments.

\section{References}

1 Tavano L, Alfano P, Muzzalupo R, de Cindio B. Niosomes vs microemulsions: New carriers for topical delivery of Capsaicin. Colloids Surf B Biointerfaces 2011; 87: 333-9.

2 Huang YB, Lin YH, Lu TM, Wang RJ, Tsai YH, Wu PC. Transdermal delivery of capsaicin derivative-sodium nonivamide acetate using microemulsions as vehicles. Int J Pharm 2008; 349: 206-11.

3 Lee SH, Richardson RL, Dashwood RH, Baek SJ. Capsaicin represses transcriptional activity of $\beta$-catenin in human colorectal cancer cells. J Nutr Biochem 2012; 23: 646-55.

4 Mori A, Lehmann S, O’Kelly J, Kumagai T, Desmond JC, Pervan M, et al. Capsaicin, a component of red peppers, inhibits the growth of androgen-independent, p53 mutant prostate cancer cells. Cancer Res 2006; 66: 3222-9.

5 Zamani S, Khoee S. Preparation of core-shell chitosan/PCL-PEG triblock copolymer nanoparticles with $A B A$ and $B A B$ morphologies: Effect of intraparticle interactions on physicochemical properties. Polymer 2012; 53: 5723-36.

6 Lu F, Lei L, Shen YY, Hou JW, Chen WL, Li YG, et al. Effects of amphiphilic PCL-PEG-PCL copolymer addition on 5-fluorouracil release from biodegradable PCL films for stent application. Int J Pharm 2011; 419: 77-84.

7 Tanaka K, Kanazawa T, Shibata Y, Suda Y, Fukuda T, Takashima Y, et al. Development of cell-penetrating peptide-modified MPEG-PCL diblock copolymeric nanoparticles for systemic gene delivery. Int J Pharm 2010; 396: 229-38.

8 Endres TK, Beck-Broichsitter M, Samsonova O, Renette T, Kissel TH. Self-assembled biodegradable amphiphilic PEG-PCL-IPEI triblock copolymers at the borderline between micelles and nanoparticles designed for drug and gene delivery. Biomaterials 2011; 32: 772131.

9 Wilson DR, Zhang N, Silvers AL, Forstner MB, Bader RA. Synthesis and evaluation of cyclosporine A-loaded polysialic acid-polycaprolactone micelles for rheumatoid arthritis. Eur J Pharm Sci 2014; 51: 146-56.

10 Zhang $\mathrm{H}$, Wilson J, Zhang J, Luo Y. Characterization of potential degradation products in a PEGylating reagent $20 \mathrm{kDa}$ monomethoxy polyethylene glycol propionaldehyde by RP-HPLC, APCI-MS and NMR. J Pharm Biomed Anal 2014; 89: 221-6.

11 Jiang X, Xin H, Sha X, Gu J, Jiang Y, Law K, et al. PEGylated poly(trimethylene carbonate) nanoparticles loaded with paclitaxel for the treatment of advanced glioma: In vitro and in vivo evaluation. Int J Pharm 2011; 420: 385-94.

12 Zhang Y, Zhang Q, Zha L, Yang W, Wang C, Jiang X, et al. Preparation, characterization and application of pyrene-loaded methoxy poly(ethylene glycol)-poly(lactic acid) copolymer nanoparticles. Colloid Polym Sci 2004; 282: 1323-8.

13 Han HS, Lee J, Kim HR, Chae SY, Kim M, Saravanakumar G, et al. 
Robust PEGylated hyaluronic acid nanoparticles as the carrier of doxorubicin: Mineralization and its effect on tumor targetability in vivo. J Control Release 2013; 168: 105-14.

14 Wei K, Peng X, Zou F. Folate-decorated PEG-PLGA nanoparticles with silica shells for capecitabine controlled and targeted delivery. Int $J$ Pharm 2014; 464: 225-33.

15 Parmar N, Singla N, Amin S, Kohli K. Study of cosurfactant effect on nanoemulsifying area and development of lercanidipine loaded (SNEDDS) self nanoemulsifying drug delivery system. Colloids Surf B Biointerfaces 2011; 86: 327-38.

16 Li TSC, Yawata T, Honke K. Efficient siRNA delivery and tumor accumulation mediated by ionically cross-linked folic acidpoly(ethylene glycol)-chitosan oligosaccharide lactate nanoparticles: For the potential targeted ovarian cancer gene therapy. Eur J Pharm Sci 2014; 52: 48-61.

17 Mohanty C, Sahoo SK. The in vitro stability and in vivo pharmacokinetics of curcumin prepared as an aqueous nanoparticulate formulation. Biomaterials 2010; 31: 6597-611.

18 Das RK, Kasoju N, Bora U. Encapsulation of curcumin in alginatechitosan-pluronic composite nanoparticles for delivery to cancer cells. Nanomedicine 2010; 6: 153-60.

19 De Baere S, Eeckhaut V, Steppe M, De Maesschalck C, De Backer P, Van Immerseel F, et al. Development of a HPLC-UV method for the quantitative determination of four short-chain fatty acids and lactic acid produced by intestinal bacteria during in vitro fermentation. J Pharm Biomed Anal 2013; 80: 107-15.

20 Yu JN, Zhu Y, Wang L, Peng M, Tong SS, Cao X, et al. Enhancement of oral bioavailability of the poorly water-soluble drug silybin by sodium cholate/phospholipid-mixed micelles. Acta Pharmacol Sin 2010; 31: 759-64.

21 Chu C, Tong SS, Xu Y, Wang L, Fu M, Ge YR, et al. Proliposomes for oral delivery of dehydrosilymarin: preparation and evaluation in vitro and in vivo. Acta Pharmacol Sin 2011; 32: 973-80.

22 Uppoor VRS. Regulatory perspectives on in vitro (dissolution)/in vivo (bioavailability) correlations. J Control Release 2001; 72: 127-32.

23 Xie W, Zhu W, Shen Z. Synthesis, isothermal crystallization and micellization of mPEG-PCL diblock copolymers catalyzed by yttrium complex. Polymer 2007; 48: 6791-8.

24 Cai R, Li R, Qian J, Xie A, Nie K. The morphology and fabrication of nanostructured micelle by a novel block copolymer with lineardendritic structure. Mater Sci Eng C Mater Biol Appl 2013; 33: 2070-7.
25 Zi P, Yang X, Kuang H, Yang Y, Yu L. Effect of HPßCD on solubility and transdermal delivery of capsaicin through rat skin. Int J Pharm 2008; 358: 151-8.

26 Shah V, Midha K, Findlay JA, Hill H, Hulse J, McGilveray I, et al. Bioanalytical method validation - a revisit with a decade of progress. Pharm Res 2000; 17: 1551-7.

27 Chakraborty S, Shukla D, Mishra B, Singh S. Lipid - An emerging platform for oral delivery of drugs with poor bioavailability. Eur J Pharm Biopharm 2009; 73: 1-15.

28 Lu Y, Kim S, Park K. In vitro-in vivo correlation: Perspectives on model development. Int J Pharm 2011; 418: 142-8.

29 Dash TK, Konkimalla VB. Poly-€-caprolactone based formulations for drug delivery and tissue engineering: A review. J Control Release 2012; 158: 15-33.

30 Xing F, Cheng G, Yi K, Ma L. Nanoencapsulation of capsaicin by complex coacervation of gelatin, acacia, and tannins. J Appl Polymer Sci 2005; 96: 2225-9.

31 Tyagi P, Chancellor MB, Li Z, de Groat WC, Yoshimura N, Fraser MO, et al. Urodynamic and immunohistochemical evaluation of intravesical capsaicin delivery using thermosensitive hydrogel and liposomes. J Urol 2004; 171: 483-9.

32 Rao JP, Geckeler KE. Polymer nanoparticles: Preparation techniques and size-control parameters. Prog Polymer Sci 2011; 36: 887-913.

33 Zhang Z, Feng SS. The drug encapsulation efficiency, in vitro drug release, cellular uptake and cytotoxicity of paclitaxel-loaded poly(lactide)-tocopheryl polyethylene glycol succinate nanoparticles. Biomaterials 2006; 27: 4025-33.

34 Gu C, Le V, Lang M, Liu J. Preparation of polysaccharide derivates chitosan-graft-poly( $\varepsilon$-caprolactone) amphiphilic copolymer micelles for 5-fluorouracil drug delivery. Colloids Surf B Biointerfaces 2014; 116: 745-50.

35 Danhier F, Feron O, Préat V. To exploit the tumor microenvironment: Passive and active tumor targeting of nanocarriers for anti-cancer drug delivery. J Control Release 2010; 148: 135-46.

36 Bernabeu E, Helguera G, Legaspi MJ, Gonzalez L, Hocht C, Taira C, et al. Paclitaxel-loaded PCL-TPGS nanoparticles: In vitro and in vivo performance compared with Abraxane ${ }^{\circledR}$. Colloids Surf B Biointerfaces 2014; 113: 43-50.

37 Knolle E, Zadrazil M, Kovacs GG, Medwed S, Scharbert G, Schemper M. Comparison of cooling and EMLA to reduce the burning pain during capsaicin 8\% patch application: A randomized, double-blind, placebocontrolled study. Pain 2013; 154: 2729-36. 